\title{
Advances in modeling of Wolf-Rayet stars
}

\author{
D. John Hillier \\ Department of Physics and Astronomy, University of Pittsburgh, \\ 3941 O'Hara Street, Pittsburgh, PA 15260, USA
}

\begin{abstract}
Due to advances in computer power and numerical techniques nonLTE line-blanketing calculations for Wolf-Rayet (WR) stars are now routine. The incorporation of blanketing has led to significant improvements in spectral analyses, and to a systematic increase in the derived WR luminosities. To make further progress we need to understand the distribution, structure, and strength of inhomogeneities in the stellar wind, and in turn, how these influence diagnostics of WR stellar parameters, and radiative driving. Further, we need to understand the physical process that initiates mass loss in WR stars. Problems with existing wind calculations are examined, and the difficulty of observationally determining the shape of the velocity law around the sonic point is discussed. To determine the wind dynamics around the sonic point, it is essential to include ions with ionization potentials in excess of $300 \mathrm{eV}$. A recent study of the O7 $\mathrm{Iaf}^{+}$ star AV 83 is discussed. The analysis indicates the existence of a clumped wind, and a relatively slow wind acceleration with the velocity law characterized by $\beta=2$. The importance of extreme Of stars, for understanding both WR and Otype star winds, is stressed. Spectra of such stars show numerous photospheric and wind features, allowing the entire wind to be probed observationally.
\end{abstract}

\section{Introduction}

The construction of theoretical Wolf-Rayet (WR) spectra is computationally demanding, even with the simplifying assumptions of the standard model (spherical geometry, prescribed mass-loss and velocity law, stationarity, homogeneity, and radiative equilibrium). Fortunately, with the advent of new computational techniques and faster computers, the major limitation with the calculation of the standard model has been removed. Non-LTE line-blanketing calculations are now routine (e.g., Hillier \& Miller 1998, 1999; Herald, Hillier \& Schulte-Ladbeck 2001; Gräfener, Koesterke \& Hamann 2002; Crowther et al. 2002a).

The new blanketed calculations have provided improved spectral fits (leading to increased confidence in the results), better ionizing fluxes for nebular calculations (e.g., Crowther et a!. 1999), and the ability to derive abundances for many different atomic species. Importantly, it is now possible to model the UV iron spectrum (e.g., Herald et al. 2001). In general, the deduced stellar luminosities have increased (see Crowther, these proceedings) which has important implications for stellar evolution, and for the WR wind 'momentum' problem.

Herald et al. (2001) performed extensive non-LTE line-blanketed calculations in order to analyse the WN8 star WR 40 (HD 96548). The most complete model included $\mathrm{He}, \mathrm{C}, \mathrm{N}, \mathrm{O}, \mathrm{Mg}, \mathrm{Al}, \mathrm{Si}, \mathrm{S}, \mathrm{Ar}, \mathrm{Ca}$ and Fe. Typical models had 
over 500 super-levels, 1800 full levels, 60 depth points and 20000 transitions. Considerably larger models can now be run, with additional ionization stages, as well as additional species such as $\mathrm{Cr}, \mathrm{Mn}$, and $\mathrm{Ni}$, included. The largest complete model run to date has 18 atomic species, approximately 100 ionization states, over 2900 super-levels, 14800 full levels, and 40 depth points.

The improved blanketed calculations do not significantly affect the spectral analysis, although the strengths of some spectral lines are changed. For spectral analysis the inclusion of $\mathrm{Fe}$ blanketing is crucial, while other species are (generally) less important. However, it should be stressed that many of the other species (e.g., S, Ar) are crucial for determining the line force. Similar improved calculations have also been performed for the WC5 star WR 111 (HD 165763, Hillier \& Lanz 2001). In general the newer calculations show excellent agreement with the older calculations, although the new model gives improved fits to the C III $\lambda 5696$ and CIV $\lambda 5805$ classification lines.

Despite recent progress, there are still significant improvements that be can be made to spectral analyses using existing codes. Better atomic data are needed, notwithstanding the enormous (and appreciated) efforts of Bob Kurucz (Kurucz 1993 \& private communication), the opacity Project (Seaton 1987), the Iron Project (Hummer et al. 1993), and others. There is little doubt that some, but certainly not all, of the errors in matching current models with observation are due to errors in the atomic data.

WR stars, both massive Pop. I WR stars and low mass Pop. II [WR] stars, can be used to address the accuracy of the atomic data. The [WC10] star CPD$56^{\circ} 8032$ (studied extensively by De Marco, Barlow \& Storey 1997a; De Marco, Storey \& Barlow, 1997b; De Marco \& Crowther 1998) is an excellent candidate to address accuracy of the atomic data. As the wind's terminal velocity is only $225 \mathrm{~km} \mathrm{~s}^{-1}$, it is possible to resolve many more lines, allowing a much more rigorous comparison between model and observation. In addition, it is possible to directly observe CiI di-electronic lines (Barlow \& Storey 1993; De Marco et al. 1997b). In principle, accurate models for this star, and similar stars, will greatly improve our understanding of $\mathrm{C}$ and $\mathrm{O}$ spectra, and hence improve our modeling of WC stars in general.

In addition to improved atomic data, more species and more lines need to be included in blanketing calculations. This is important for understanding line-driving, and for ultimately producing a model in which the mass-loss rate and velocity law are determined self-consistently, and which gives a spectrum in agreement with observation. Finally we note that improved reddening determinations and corrections are required, a point also stressed at the previous IAU symposium (No. 193) on WR stars (Hillier 1999). Generally a standard reddening law is used, but this is not always appropriate. Further, it should be noted that the standard extinction law of Seaton (1979) differs substantially from that of Cardelli, Clayton \& Mathis (1988) shortward of $1300 \AA$. While the reddening law can be derived from the model fits, the derived reddening is not unique (e.g., it depends on $\beta$ ), and in addition it does not allow the models to be tested. Ideally, a study of O/B stars in the neighborhood of WR stars needs to be undertaken in order to accurately constrain both the reddening, and the reddening law. 


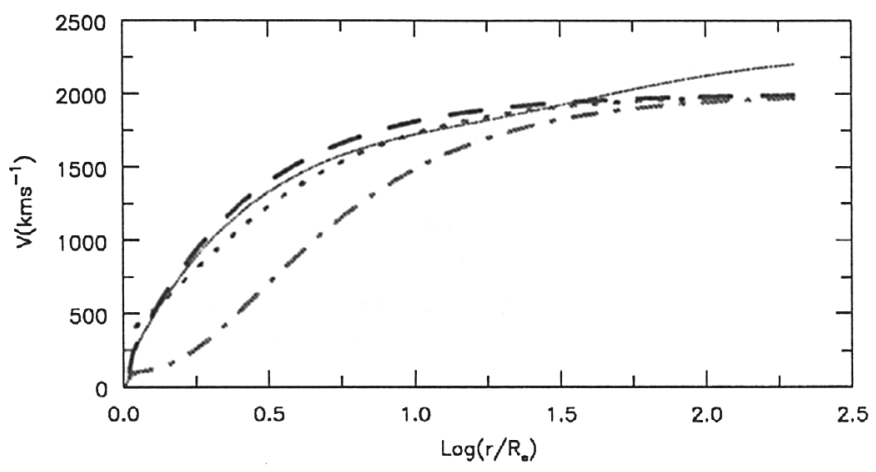

Figure 1. An illustration of several different velocity laws. Solid line: $v_{\infty}=2300 \mathrm{~km} \mathrm{~s}^{-1}, v_{\mathrm{ext}}=400 \mathrm{~km} \mathrm{~s}^{-1}, \beta_{1}=1$ and $\beta_{2}=50$. Dashed line: $\beta=1$, $v_{\infty}=2000 \mathrm{kms}^{-1}$. Dot-dashed line: $\beta=3, v_{\infty}=2000 \mathrm{~km} \mathrm{~s}^{-1}$. Dotted line: As for dot-dashed but shifted by -0.3 in $\log \left(r / R_{*}\right)$. Note the similarity between the $\beta=1$ law and the shifted $\beta=3$ law for $v>500 \mathrm{~km} \mathrm{~s}^{-1}$.

\section{The velocity law}

For most WR spectral analyses a standard $\beta$-velocity law $v(r)=\left(1-R_{*} / r\right)^{\beta}$ with $\beta=1$ has been adopted. However, in the literature there are numerous studies which suggest that $\beta$ is significantly greater than 1 . Fortunately, the derived WR luminosities are not strongly influenced by $\beta$. From analysis of line-profile variability, Lépine \& Moffat (1999) deduced a high value of $\beta$ (>3), although this conclusion has been questioned by Koesterke, Hamann \& Urrutia (2001). More recently Antokhin \& Cherepashchuk (2001) deduced $\beta>1$, and found departures from the normal $\beta$-velocity law, from analysis of eclipse observations of the WN5+O6III-V binary V444 Cygni (WR 139).

It is important to note that $\beta$ is a single parameter which was devised to characterize the velocity law in O-type star winds based on the predictions of radiation driven wind theory. In the dense winds of WR stars there is no reason to expect that such a simple prescription should still hold. Further, the use of a single parameter cannot simultaneously describe changes in the velocity near the sonic point, and changes in the shape of the velocity law in the outer region.

To accommodate a wider range of velocity law, Hillier proposed a velocity law of the form

$$
v(r)=\frac{v_{\mathrm{o}}+\left(v_{\infty}-v_{\mathrm{ext}}-v_{\mathrm{o}}\right)\left(1-R_{*} / r\right)^{\beta_{1}}+v_{\mathrm{ext}}\left(1-R_{*} / r\right)^{\beta_{2}}}{1+\left(v_{\mathrm{o}} / v_{*}-1\right) \exp \left[\left(R_{*}-r\right) / h_{\mathrm{eff}}\right]}
$$

The denominator is used to give an exponential scale height at depth, and can be ignored for the present discussion (note that the denominator in the expression of Hillier \& Miller [1999] contained typographical errors). Assuming $v_{0}$ to be small $\left(\ll v_{\infty}\right)$, the second component of the numerator describes the acceleration in the inner wind, while the third component affects the shape of the velocity law in the line forming region. For WR 111 (HD 165763, WC5), Hillier \& Miller (1999) found that $v_{\infty}=2300 \mathrm{~km} \mathrm{~s}^{-1}, v_{\text {ext }}=400 \mathrm{~km} \mathrm{~s}^{-1}, \beta_{1}=1$ and $\beta_{2}=50$ (see Figure 1) gave a reasonable match to the spectrum. However it must be noted that $\beta_{2}$ and $v_{\text {ext }}$ were poorly constrained. Several different velocity laws are illustrated in Figure 1. 
Hillier (1991a) showed, using a model for a WN5 star, that it was very difficult to distinguish spectroscopically between different $\beta$ velocity laws. This occurs because (generally) the inner wind (say $v(r)<v_{\infty} / 4$ ) is optically thick, and only the outer wind can be constrained via spectroscopic modeling. More recent calculations using $\beta=1, \beta=2$, and $\beta=3$ (see Figure 1) verify this conclusion, for both WN8 stars, and WC4/5 stars. In general, when modeling WR stars, a larger (assumed) value of $\beta$ (actually $\beta_{1}$ ) leads to a smaller core radius, $R_{*}$. While there are some differences in line strengths and continuum shapes, the differences are relatively minor and can be at least partially compensated by changes in stellar parameters and in the adopted extinction.

\section{Improvements}

To improve modeling of WR stars progress is needed in two key areas. These are determining $\dot{M}$ and $v(r)$ self-consistently from the radiation field, and relaxing the assumption of homogeneity.

\subsection{The dynamics of Wolf-Rayet winds}

Despite the advances in constructing non-LTE line-blanketed models, no selfconsistent line-driven WR wind model has been calculated. For WR stars, unlike O-type stars, the wind momentum generally exceeds the single scattering limit, often by a large factor. The ratio of the wind momentum to the single scattering limit $\left[=\dot{M} v_{\infty} /(L / c)=\eta\right]$ is termed the performance number (Springmann 1994). Values of $\eta>1$ indicate the importance of multiple scattering. Fortunately, detailed non-LTE line-blanketing calculations and the inclusion of clumping have reduced 'observed' values of $\eta$ to (generally) less than 10 (Hamann \& Koesterke 2000; Crowther et al. 2002a). As discussed below, such values are, in principle, explainable by radiation driven wind theory.

Lucy \& Abbott (1993) showed the importance of the variable ionization structure, which enhances photon trapping, for line driving. Using a MonteCarlo approach they were able to produce models in which $\dot{M}$ exceeded the single scattering limit by a factor of 10 (i.e., $\eta=10$ ). While they were able to obtain a global consistency for the line force, their model did not work in the deeper layers. No quantitative comparison between observation and model was performed. Their models also neglected clumping, now believed to be of crucial importance in explaining WR spectra. Springmann (1994) also undertook detailed Monte-Carlo investigations to examine momentum deposition in the dense winds of WR stars. Springmann found that performance numbers greater than unity were possible provided there were sufficient overlapping lines.

Schmutz (1997) attempted to compute self-consistent models for the WR star WR 6 (EZ CMa, HD 50896, WN4). Like Lucy \& Abbott, Schmutz found that his models had insufficient line force in the deeper layers. Schmutz noted that opacity must be missing from his models, since his opacities in the inner wind were lower than the OPAL Rosseland mean opacities adopted in evolutionary calculations. Key ingredients of Schmutz's models were the assumption of photon loss in the He II Ly $\alpha$ line, and the inclusion of clumping. These allowed Schmutz to obtain a model in which the single scattering limit was exceeded by a factor of six, and which could match the observed He II and He I spectrum of WR 6 . 


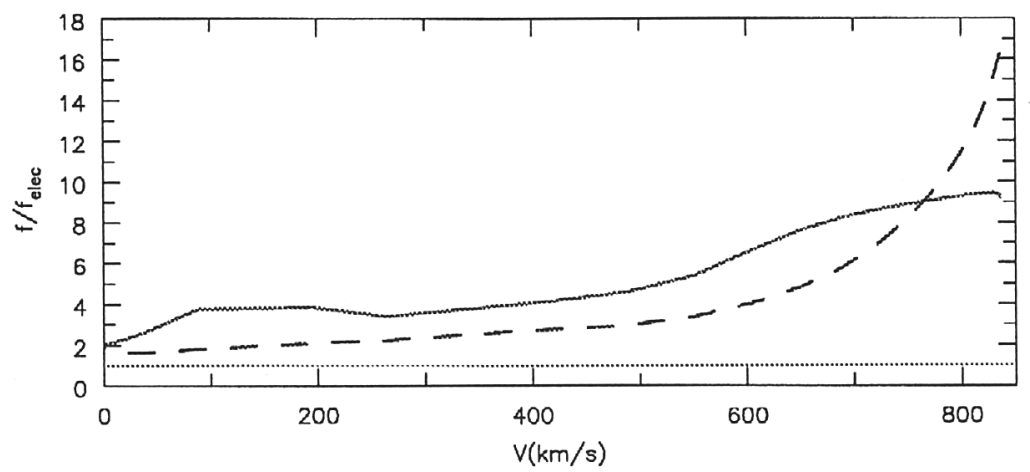

Figure 2. Illustration of the variation of $f_{\text {rad }} / f_{\text {elec }}$ (heavy solid line) with velocity in a model for WR 40 (WN8) with $L=7.5 \times 10^{5} \mathrm{~L}_{\odot}$, and $\beta=1$. $f_{\mathrm{rad}}$ is the total radiation force, while $f_{\text {elec }}$ is the radiation force due to electron scattering only. Also shown (dashed line) is $\left(v d v / d r+\rho^{-1} d P / d r+g\right) / f_{\text {elec }}$ calculated assuming $M=40 \mathrm{M}_{\odot}$. In the outer layers $\left(v>0.9 v_{\infty}\right)$ the radiation force exceeds that needed to drive the wind. In intermediate layers $\left(v \simeq v_{\infty} / 2\right)$ the radiation force in insufficient. However the discrepancy is not large (line force needs to be increased by less than a factor of 2) and the discrepancy might be removed by changes in the velocity law or volume filling factor, and by improvements in the model (more species, more lines, better atomic models). At smaller velocities $\left(e . g ., 100 \mathrm{~km} \mathrm{~s}^{-1}\right)$ the discrepancy is significantly larger. Tests to date have not revealed a solution to this discrepancy. Models with $\beta=2$ show less of a discrepancy in this region, but it is still significant.

Gayley, Owocki \& Cranmer (1995) considered the grey model, allowing them to gain analytical insight into the effects of multiple scattering. As stressed by Gayley et al. , the principal problem in driving WR winds is finding sufficient opacity - it is not a momentum problem. A more detailed discussion of the above issues is given by Owocki \& Gayley (1999).

With the advent of line-blanketed models it was hoped that detailed dynamic models could be constructed. While the models can drive the winds in the outer layer they fail around the sonic point (Figure 2). A problem in understanding WR dynamics arises because of the importance of clumping. As the clumping factor is not easily deduced, mass-loss rates for WR stars have considerable uncertainty. Further, it is unclear whether the simple filling factor approach allows an accurate radiation force to be computed.

One of the problems with many models is that they do not include sufficiently high ionization stages. Even for WN8 stars, species with ionization potentials (IPs) of the order of $125 \mathrm{eV}$ must be included in the models (Figure 3). If $\beta$ is significantly greater than 1 (e.g., $\beta=3$ ) even higher ionization stages need to be included (with IPs over $200 \mathrm{eV}$ ). For the luminous WC4 stars in the LMC the situation is even worse. For such stars it may be necessary to include ionization stages with IPs up to $400 \mathrm{eV}$. For Fe this means over 10 ionization stages of $\mathrm{Fe}$ (Fe IV to Fe XIV) must be included if the entire wind is to be modeled. Because the winds of WR stars are optically thick, the higher ionization stages do not significantly influence the spectral analyses, but do sub- 


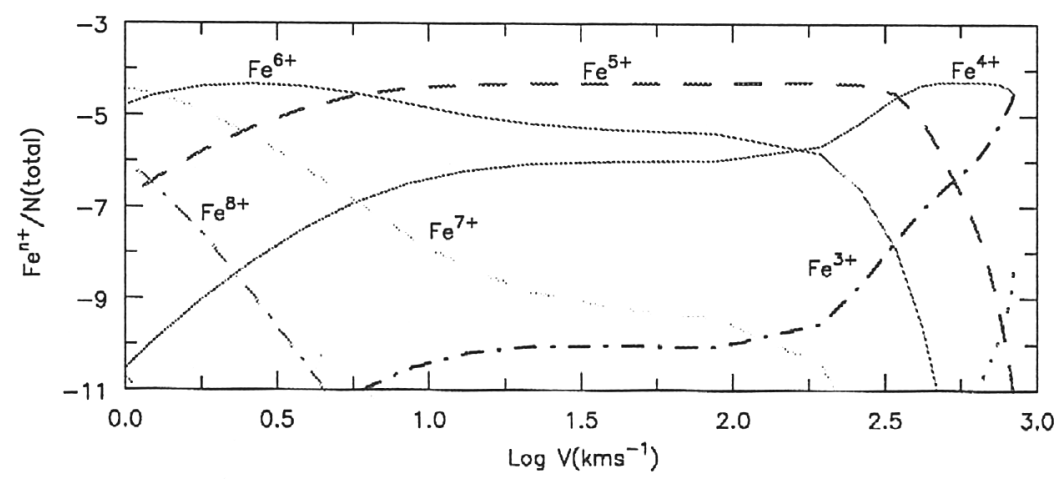

Figure 3. Illustration of the Fe ionization structure as a function of velocity in a model for WR 40 (WN8) assuming a $\beta=1$ velocity law. If $\beta=3$, the dominant ionization stage at low velocities is shifted by +2 (i.e., $\mathrm{Fe}^{9+}(\mathrm{IP}=262 \mathrm{eV})$ becomes the dominant ion at $1 \mathrm{~km} \mathrm{~s}^{-1}$, while $\mathrm{Fe}^{7+}(\mathrm{IP}=151 \mathrm{eV})$ is dominant at $10 \mathrm{~km} \mathrm{~s}^{-1}$ ).

stantially modify the structure of the inner wind and the radiation force in that region.

More recently, Nugis \& Lamers (2002) have examined an optically thick radiation driven wind. They argue for the importance of the opacity maximum seen in the OPAL data (Iglesias \& Rogers 1996) in determining the structure of WR winds. They further argue that the opacity of WR winds must increase at the sonic point, and this restricts the temperature at the sonic point to be near $160000 \mathrm{~K}$, or to lie between 40000 and $70000 \mathrm{~K}$. They further argue that this can only be achieved by winds with a very large $\beta(\approx 5)$.

In support of their claims, Nugis \& Lamers (2002) note that Hamann \& Schwarz (1992) derived a very large radius for the WN5 star in V444 Cygni. This argument is no longer valid since Hamann \& Schwarz used unclumped models. Models with clumping can match the spectrum of V444 Cygni using much smaller core radii (e.g., Kurosawa, Hillier \& Pittard 2002). However, it is generally true that radii derived from the standard model exceed stellar evolution radii, although it is also true that as the standard model has improved the derived radii have been reduced.

\subsection{Clumping}

It is now generally accepted that WR winds are clumped. First, clumping is predicted by radiation driven wind theory (e.g., Owocki et al. 1988). Second, clumping is required to explain the observed line profile variability (e.g., Robert 1994; Lépine, Moffat \& Henriksen 1996; Lépine \& Moffat 1999). Third, clumping is required to explain the observed weakness of electron scattering wings in WR stars (e.g., Hillier 1991b), and the velocity shift and shape of the C IV $\lambda 1550$ doublet profile in WC stars (Crowther et al. 2002a).

In detailed spectral calculations a standard filling factor approach is used to include clumping. In this approach there are typically two free parameters the volume filling factor, and the velocity at which clumping becomes important (e.g., Hillier \& Miller 1999). The interclump medium is ignored. This approach 
appears to work surprisingly well, and provides spectra in much better agreement (as compared to non-clumped models) with observation.

Several investigations have also been made of the influence of clumping on the infrared/radio energy distribution. The basic parameters of these models are the volume filling factor and the density contrast (Abbott, Bieging \& Churchwell 1981; Lamers \& Waters 1984). A recent investigation of Nugis, Crowther \& Willis (1998) found that (generally) clumping must be more important in the IR (rather than radio) formation region.

Despite the success of the filling factor approach it is very simplistic. It assumes that the clumps are identical, that there is no interclump medium (for the transfer models), and ignores radiative transfer effects related to individual clumps. It also assumes that the clumps are small relative to the photon mean free-path, which is valid for continuum processes, but not for line processes where the clumps are expected to have a size similar to the Sobolev length.

A distribution of clump sizes might enhance the emission from low ionization stages, since emission from such species would tend to come from the densest clumps. Since ionization ratios are used to provide constraints on stellar luminosities, current WR luminosities could still be underestimated. Some basis for believing a range of clump sizes exists in WR winds is provided by the difficulty of matching lines belonging to the lowest ionization stages (e.g., $\mathrm{C} \mathrm{II}, \mathrm{He}$ ) and the highest ionization stages (e.g., $\mathrm{N} \mathrm{V}, \mathrm{O} \mathrm{VI})$ simultaneously. It is particularly difficult to match the O VI $\lambda \lambda 3811,3834$ doublet in WC stars.

Radiative transfer effects in clumped media can be very complex (e.g., Williams 1992; Hillier 1996). In particular, the assumption of spherical symmetry is lost, clumps could be optically thick even to continuum radiation, and radiation may scatter around clumps. In addition, the velocity distribution of clumps will affect line profiles.

Since clumping introduces free-parameters, it is important to obtain additional probes into their nature. Polarization and line variability studies offer such probes. Recently Dessart \& Owocki (2002) used hydrodynamical simulations and a 'patch' approach to model optically thin lines in WR stars. An interesting conclusion of their work is that the angular patch size of $3^{\circ}$ can explain the observed profile variations.

\section{O-type star modeling}

O-type stars and WR stars are often treated and discussed separately, however they form a sequence in wind properties. The most extreme O-type stars provide a key to further our understanding of both WR and O-type star winds. Unlike the majority of O-type stars, the most extreme O-type stars contain numerous diagnostics of the stellar wind. Unlike WR stars, the wind is not thick, and photospheric features can still be seen. Thus the extreme O-type stars show spectral features which allow us to probe both the photosphere and the entire wind.

As part of a more general program, we (Hillier, Lanz, Heap, Hubeny, Smith, Evans, Lennon) have modeled the SMC stars AV 83 (Of7 Iaf) and AV 69 (OC7.5 III ((f)) ) (Hillier et al. 2003). The stars have very similar effective temperatures $(\sim 34000 \mathrm{~K})$ and luminosities $\left(\sim 3.5 \times 10^{5} \mathrm{~L}_{\odot}\right)$ but show very different 
wind spectra. The wind of AV 83 is significantly denser than for AV 69. Of immediate interest is, why do the stars show different wind spectra?

From our analysis we concluded that AV 83 is in a much more advanced evolutionary state than AV 69. In particular, AV 83 shows evidence for both He- and N-enrichment, and it has a lower effective gravity than does AV 69. In support of the lower $g_{\text {eff }}$ for AV 83, we note that $v_{\infty} \simeq 950 \mathrm{~km} \mathrm{~s}^{-1}$, while for AV $69 v_{\infty} \simeq 1800 \mathrm{~km} \mathrm{~s}^{-1}$. Interestingly, the velocity law in the wind of AV 83 can be characterized by $\beta=2$, indicating a much slower velocity law than normal for O-type stars. This may have implications for the denser winds of WR stars. As for WR stars we also find evidence that the wind of AV 83 is clumped. Such evidence comes from analysis of the PV profiles (see Crowther et al. 2002b), and from the blue edges of strong photospheric lines. Further, the model had difficulty driving the flow by radiation pressure near the sonic point. A major issue related to this difficulty is whether microturbulence (required to fit strong photospheric lines) has to be included in the computation of the line force.

\section{Summary}

By the next IAU Symposium on hot massive stars, the current generation of models should provide us with a much improved understanding of both WR and O-type star stellar winds. In addition, the mass-loss process in WR stars will be much better understood. Unfortunately, progress in computing synthetic spectra for highly inhomogeneous models will be much more difficult to accomplish.

Acknowledgments. The author gratefully acknowledges partial support from NASA grant NAGW-3828.

\section{References}

Abbott, D.C., Bieging, J.H., Churchwell, E. 1981, ApJ 250, 645

Antokhin. I.I., Cherepashchuk, A.M. 2001, Astron. Zh. 78, 313 (= Astron. Reports 45, 269)

Barlow, M.J., Storey P.J. 1993, in: R. Weinberger \& A. Acker (eds.), Planetary Nebulae, Proc. IAU Symp. No. 155 (Dordrecht: Kluwer), p. 105

Cardelli, J.A., Clayton, G.C., Mathis, J.S. 1988, ApJ (Letters) 329, L33

Crowther, P.A., Pasquali, A., De Marco, O., et al. 1999, A\&A 350, 1007

Crowther, P.A., Dessart, L., Hillier, D.J., et al. 2002a, A\&A 392, 653

Crowther, P.A., Hillier, D.J. Evans, C.J., et al. 2002b, ApJ 579, 774

De Marco, O., Barlow, M.J., Storey, P.J. 1997a, MNRAS 292, 86

De Marco, O., Storey, P.J., Barlow, M.J. 1997b, MNRAS 297, 999

De Marco, O., Crowther, P.A. 1998, MNRAS 296, 419

Dessart, L., Owocki, S.P. 2002, A\&A 383, 1112

Gayley, K.G., Owocki, S.P., Cranmer, S.R. 1995, ApJ 442, 296

Gräfener, G., Koesterke, L., Hamann W.-R. 2002, A\&A 387, 244

Hamann, W.-R., Schwarz, E. 1992, A\&A 261, 523

Hamann, W.-R., Koesterke, L. 2000, A\&A 360, 647

Herald, J.E., Hillier, D.J., Schulte-Ladbeck, R.E. 2001, ApJ 548, 932

Hillier, D. J. 1991a, in: L. Crivellari, I. Hubeny \& D. G. Hummer (eds.), Stellar Atmospheres: Beyond Classical Models, NATO ASI Ser. Vol. 341 (Dordrecht: Kluwer), p. 317 
Hillier, D.J. 1991b, A\&A 247, 455

Hillier, D.J. 1996, in: J.-M. Vreux, A. Detal, D. Fraipont-Caro, E. Gosset \& G. Rauw (eds.), Wolf-Rayet Stars in the Framework of Stellar Evolution, Proc. 33rd Liege Int. Astrophys. Coll. (Liége:Université de Liége), p. 509

Hillier, D.J. 1999, in: K.A. van der Hucht, G. Koenigsberger \& P.R.J. Eenens (eds.), Wolf-Rayet Phenomena in Massive Stars and Starburst Galaxies, proc. IAU Symp. No. 193 (San Francisco: ASP), p. 129

Hillier, D.J., Miller, D.L. 1998, ApJ 496, 407

Hillier, D.J., Miller, D.L. 1999, ApJ 519, 354

Hillier, D.J., Lanz, T. 2001, in: G. Ferland \& D.W. Savin (eds.), Spectroscopic Challenges of Photoionized Plasmas, ASP-CS 247, 343

Hillier, D.J., Lanz, T., Heap, S.R., et al. 2003, ApJ in press

Hummer, D.G., Berrington, K.A., Eissner, A., et al. 1993, A\&A 279, 298

Iglesias, C.A., Rogers, F.J., 1996, ApJ 464, 943

Koesterke, L, Hamann, W.-R., Urrutia, T. 2001, A\&A 379, 224

Kurosawa, R., Hillier, D.J., Pittard, J.M. 2002, A\&A 388, 957

Kurucz, R.L. 1993, CD-ROM 22, Atomic Data for Fe and Ni (Cambridge: SAO)

Lamers, H., Waters, L.B.F.M. 1984, A\&A 138, 25

Lépine, S., Moffat, A.F.J., Henriksen, R.N. 1996, ApJ 466, 392

Lépine, S., Moffat, A.F.J. 1999, ApJ 514, 909

Lucy, L.B., Abbott, D.C. 1993, ApJ 405, 738

Nugis, T., Crowther, P.A., Willis, A.J. 1998 A\&A 333, 956

Nugis, T., Lamers, H. 2002, A\&A 389, 162

Owocki, S.P., Castor, J.I., Rybicki, G.B. 1988, ApJ 335, 914

Owocki, S.P., Gayley, K.G. 1999, in: K.A. van der Hucht, G. Koenigsberger \& P.R.J. Eenens (eds.), Wolf-Rayet Phenomena in Massive Stars and Starburst Galaxies, IAU Symp. No. 193 (San Francisco: ASP), p 157

Robert, C. 1994, in: A.F.J. Moffat, S.P. Owocki, A.W. Fullerton, N. St-Louis (eds.), Instability and Variability of Hot-Star Winds, Ap\&SS 221, 137

Schmutz, W. 1997, A\&A 321, 268

Seaton, M.J. 1979, MNRAS (Letters) 187, 73P

Seaton, M.J. 1987, J. Phys. B. 20, 6363

Springmann, U. 1994, A\&A 289, 505

Williams, R.E. 1992, ApJ 392, 99

\section{Discussion}

KOENIGSBERGER: Doesn't increasing the microturbulent velocity reduce the depth of the absorptions?

HILLIER: The principal effect of microturbulence is to enhance the equivalent width of strong (saturated) lines, while the equivalent width of weak lines is not affected. In O-type stars the shape of the line is generally dominated by rotation.

MASSEY: You made the cryptic comment that the reddening laws for WR stars somehow differed from the average law. Have you tried to derive this yourself from the pair method? Does it differ significantly from the OB stars in the same cluster? Maybe you could elaborate? 
HILlIER: When modeling WR stars, especially in the UV, we are forced to work in flux-space since the 'continuum' is non-existent. This has several difficulties. First, different groups use different 'average' reddening laws below $1500 \AA$. Secondly, anomalous reddening is definitely seen towards some WR stars, while other WR stars are in regions which may have an anomalous reddening law. We tried the pair method for the WN8 stars HD 86161 (WR 16) and HD 96548 (WR 40), but concluded that the stars were not sufficiently similar.

WALBORN: I'd like to emphasize the significance of the CNO abundance results in AV 83 and AV 69. The former has a 'normal' Of spectrum, meaning typical or majority, and is mixed; while I classified the latter as OC and you find normal SMC abundances. Therefore, the previous result found in late $\mathrm{O}$ - and B-type supergiants, that the normal majority have processed material in their atmospheres and winds, while the OBC minority have normal abundances, is re-affirmed, perhaps for the first time in this spectraltype range. This result, along with the essentially identical H-R diagram locations of these two stars, might be explained if AV 83 had a lower initial mass and higher initial rotational velocity, thus mixing and moving upward in the $\mathrm{H}-\mathrm{R}$ diagram as predicted by evolutionary tracks with rotation; while AV 69 followed a classical horizontal track; unmixed, to the same current H-R diagram location. Alternatively, AV 83 might be a product of binary evolution.

HILliER: Thank you for these illuminating comments. We concluded that AV 83 had a higher rotational velocity.

CherepashChUK: I have some comment. Recently, our group has re-determined the velocity law for V444 Cyg (WR 139, WN5+O6III-V) from a light curve solution. The empirical value of $\beta$ is $3-5$, thus much larger than unity.

HILliER: This is an interesting result, and it would be useful to run a model for V444 Cyg with your velocity law. Our experience with modeling V444 Cyg is that, it is difficult to get firm constraints, because of the unknown luminosity ratio of the component stars, and because the stellar parameters derived from light curve fits are extremely sensitive to the inclination of the binary system.

Puls: You mentioned the problem with the OVI line. Can't the X-rays help in the same way as is the case in O-type stars?

HILlIER: The X-rays can help with the formation of the $\mathrm{O}$ VI resonance line, but appear to have little effect on the O VI doublet near $3820 \AA$. However, it should be noted that the winds of most WR stars are optically thick to X-rays. Consequently we don't have any good observational constraints on the amount of X-rays produced in the accelerating region of the flow.

ANONYMOUS: John, what are the error bars in your O-type models for the He abundance?

HILlIER: We did not determine meaningful errors for the $\mathrm{H} / \mathrm{He}$ abundance, because the errors are dominated by systematic effects (e.g., microturbulence), and because in AV 83 we still have some inconsistencies in the fitting. However, I do believe the enhanced He abundance in AV 83 relative to AV 69 is real. 